\title{
Opioid-free anesthesia within an enhanced recovery after surgery pathway for minimally invasive lumbar spine surgery: a retrospective matched cohort study
}

\author{
Ellen M. Soffin, MD, PhD, 1,2 Douglas S. Wetmore, MD,1,2 James D. Beckman, MD,, \\ Evan D. Sheha, MD, ${ }^{3}$ Avani S. Vaishnav, MBBS, ${ }^{3}$ Todd J. Albert, MD, ${ }^{3,4}$ Catherine H. Gang, MPH, ${ }^{3}$ \\ and Sheeraz A. Qureshi, MD,3
}

'Department of Anesthesiology, Critical Care \& Pain Management, Hospital for Special Surgery; 2Department of Anesthesiology, Weill Cornell Medicine; ${ }^{3}$ Department of Orthopaedic Surgery, Hospital for Special Surgery; and ${ }^{4}$ Department of Orthopaedic Surgery, Weill Cornell Medicine, New York, New York

OBJECTIVE Enhanced recovery after surgery (ERAS) and multimodal analgesia are established care models that minimize perioperative opioid consumption and promote positive outcomes after spine surgery. Opioid-free anesthesia (OFA) is an emerging technique that may achieve similar goals. The purpose of this study was to evaluate an OFA regimen within an ERAS pathway for lumbar decompressive surgery and to compare perioperative opioid requirements in a matched cohort of patients managed with traditional opioid-containing anesthesia (OCA).

METHODS The authors performed a retrospective analysis of prospectively collected data. They included 36 patients who underwent lumbar decompression under their ERAS pathway for spinal decompression between February and August 2018. Eighteen patients who received OFA were matched in a 1:1 ratio to a cohort managed with a traditional OCA regimen. The primary outcome was total perioperative opioid consumption. Postoperative pain scores (measured using the numerical rating scale [NRS]), opioid consumption (total morphine equivalents), and length of stay (time to readiness for discharge) were compared in the postanesthesia care unit (PACU). The authors also assessed compliance with ERAS process measures and compared compliance during 3 phases of care: pre-, intra-, and postoperative.

RESULTS There was a significant reduction in total perioperative opioid consumption in patients who received OFA ( $2.43 \pm 0.86$ oral morphine equivalents [OMEs]; mean \pm SEM), compared to patients who received OCA (38.125 \pm 6.11 OMEs). There were no significant differences in worst postoperative pain scores (NRS scores $2.55 \pm 0.70$ vs $2.58 \pm$ 0.73 ) or opioid consumption (5.28 \pm 1.7 vs $4.86 \pm 1.5$ OMEs) in the PACU between OFA and OCA groups, respectively. There was a clinically significant decrease in time to readiness for discharge from the PACU associated with OFA (37 minutes), although this was not statistically significantly different. The authors found high overall compliance with ERAS process measures (91.4\%) but variation in compliance according to phase of care. The highest compliance occurred during the preoperative phase $(94.71 \% \pm 2.88 \%)$, and the lowest compliance occurred during the postoperative phase of care $(85.4 \% \pm 5.7 \%)$.

CONCLUSIONS OFA within an ERAS pathway for lumbar spinal decompression represents an opportunity to minimize perioperative opioid exposure without adversely affecting pain control or recovery. This study reveals opportunities for patient and provider education to reinforce ERAS and highlights the postoperative phase of care as a time when resources should be focused to increase ERAS adherence.

https://thejns.org/doi/abs/10.3171/2019.1.FOCUS18645

KEYWORDS enhanced recovery after surgery; ERAS; opioid-free anesthesia; spine surgery; lumbar decompression; opioid epidemic

ABBREVIATIONS ASA = American Society of Anesthesiologists; ERAS = enhanced recovery after surgery; LOS = length of stay; MAC = minimum alveolar concentration; MIS = minimally invasive surgical; MMA = multimodal analgesia; NRS = numerical rating scale; OCA = opioid-containing anesthesia; OFA = opioid-free anesthesia; OIH = opioid-induced hyperalgesia; OME = oral morphine equivalent; PACU = postanesthesia care unit; PONV = postoperative nausea and vomiting.

SUBMITTED December 1, 2018. ACCEPTED January 21, 2019.

INCLUDE WHEN CITING DOI: 10.3171/2019.1.FOCUS18645. 
$\mathrm{D}$ ESPITE widespread attention to the hazards of opioid agents, opioid misuse remains a leading cause of accidental death in the US. ${ }^{27}$ For many patients with a long-term opioid misuse disorder, the first episode of opioid consumption can be traced to the perioperative period. ${ }^{6}$ These risks mandate strategies to minimize and eliminate perioperative opioid exposure wherever possible. There are multiple opportunities for the anesthesiologist, surgeon, and institution to reduce opioid exposure and minimize patient harm..$^{16,31,33}$ The best-characterized clinical strategies include the use of multimodal analgesia (MMA) and enhanced recovery after surgery (ERAS) initiatives to standardize care and improve outcomes while providing satisfactory perioperative pain control. MMA has been consistently demonstrated to minimize opioid consumption and related side effects and is considered a vital component of ERAS pathways. ${ }^{39}$ Conversely, relatively little attention has been paid to multimodal anesthetic agents and whether multimodal anesthesia may also minimize opioid requirements and promote rapid recovery after surgery.

Opioid-free anesthesia (OFA) has recently been receiving interest as a potential strategy to fill this knowledge gap. ${ }^{22,35}$ OFA is a technique in which no intraoperative opioid is administered via any route, including systemic, neuraxial, or tissue infiltration. Initially pioneered for bariatric surgery, the technique relies on combinations of nonopioid agents and adjuncts, including propofol, dexmedetomidine, lidocaine, magnesium, and ketamine to produce anesthesia, sympatholysis, and analgesia..$^{21} \mathrm{In}$ contrast to OFA, traditional anesthetic protocols rely on intraoperative opioids to achieve these 3 important surgical conditions.

Results from case reports and prospective studies are accumulating to support OFA as a tool that offers the following: 1) equivalent intraoperative anesthetic conditions compared to opioid-containing regimens, ${ }^{3,19}$ 2) improved postoperative analgesia with opioid-sparing effects, ${ }^{17,19}$ 3) shorter duration of postanesthesia care unit (PACU) length of stay (LOS), ${ }^{8} 4$ ) reduced postoperative nausea and vomiting (PONV), ${ }^{40}$ and 5) higher patient satisfaction. ${ }^{8}$ OFA has further been proposed to be especially valuable in patients at high risk of opioid-related complications, including those with chronic pain conditions, opioid misuse disorder, and obstructive sleep apnea. ${ }^{35}$

To date, there are no comprehensive reports of OFA for spine surgery. However, 2 case reports in multilevel spine fusion suggest the technique may be feasible. ${ }^{7,14} \mathrm{In}$ the present article, we report the first cohort study of OFA for spine surgery. We performed a retrospective, nested comparison of OFA to our standard (opioid-containing) anesthetic regimen within an ERAS pathway for minimally invasive lumbar decompression..$^{2}$

Thirty-six patients underwent lumbar decompression/ microdiscectomy using a minimally invasive surgical (MIS) approach according to our institutional ERAS pathway. Half of the cohort received OFA. We report a significant opioid-sparing effect when using OFA without differences in pain control or postoperative opioid consumption. When used with comprehensive multimodal analgesia within an ERAS pathway of care for lumbar decompres- sion, OFA is a feasible method for protecting patients from potential unnecessary opioid exposure.

\section{Methods}

The study was carried out with institutional IRB approval. We retrospectively analyzed data from a prospectively maintained database of patients undergoing elective lumbar decompression (laminectomy, laminotomy, and/ or microdiscectomy). We included 36 patients presenting for surgery between February 2018 and August 2018. Eighteen patients underwent surgery in which an OFA technique was used. A matched cohort of 18 patients underwent surgery in which an opioid-containing anesthesia (OCA) technique was used. Patients who received intraoperative opioids were matched with patients who did not, based on age, sex, type and extent of surgery, smoking status, American Society of Anesthesiologists (ASA) class, comorbid conditions (including hypertension, diabetes mellitus, and body mass index), and baseline opioid use (opioid naïve or tolerant; Table 1).

\section{Anesthetic Technique}

All patients were cared for under an ERAS pathway for lumbar decompression, as described previously. ${ }^{32}$ In brief, preoperatively, patients were educated on the use of the numeric rating scale (NRS) to report pain and how pain scores translated to opioid administration in the PACU (see Postoperative Care). Patients were given oral acetaminophen $(1000 \mathrm{mg})$ and gabapentin $(300 \mathrm{mg})$ in the preoperative holding area, provided they had no contraindications.

General anesthesia was administered via endotracheal intubation. On arrival to the operating room, standard ASA monitors were applied (electrocardiography, noninvasive blood pressure monitor, and pulse oximetry), and premedication was provided (midazolam $0.05 \mathrm{mg} / \mathrm{kg}$ ). A balanced intravenous crystalloid solution (lactated Ringer's solution) was administered $(8-12 \mathrm{ml} / \mathrm{kg} / \mathrm{hr})$, and anesthetic induction was performed while providing $100 \%$ oxygen $(10 \mathrm{~L} / \mathrm{min})$. All patients received propofol $(1.5-2$ $\mathrm{mg} / \mathrm{kg}$, titrated to apnea) lidocaine $(1.5 \mathrm{mg} / \mathrm{kg})$, and vecuronium $(0.1 \mathrm{mg} / \mathrm{kg})$ for induction. Patients in the OCA cohort additionally received fentanyl $(2 \mu \mathrm{g} / \mathrm{kg})$ as part of the induction regimen.

After endotracheal intubation and prone positioning, patients in the OCA cohort were given boluses of fentanyl $(1-2 \mu \mathrm{g} / \mathrm{kg}$, titrated to effect) or Dilaudid (up to $2 \mathrm{mg}$ total), according to the judgment of the anesthesiologist to achieve optimal hemodynamic and anesthetic conditions for surgery. All patients received infusions of the following: 1) propofol (50-150 $\mu \mathrm{g} / \mathrm{kg} / \mathrm{hr}$ ), adjusted to maintain the mean arterial pressure within $\pm 20 \%$ of each patient's baseline value, 2) ketamine (0.1-0.5 mg/min), and 3) lidocaine $(2 \mathrm{mg} / \mathrm{kg} / \mathrm{hr}$ until closure of the surgical incision). Inhaled halogenated agents (isoflurane or sevoflurane) were permitted, up to 0.5 minimum alveolar concentration (MAC), as needed. Mechanical ventilation was achieved with 1:1 mixture of oxygen:air $\left(\mathrm{FiO}_{2} 50 \%\right)$ with a tidal volume of $6-8 \mathrm{ml} / \mathrm{kg}$ and respiratory rate of $8-14$ titrated to an end-tidal carbon dioxide between $30-35 \mathrm{~mm}$ 
Hg. Dual antiemetic therapy with dexamethasone (4 or 8 $\mathrm{mg}$ ) and ondansetron (4 mg) were provided. Ketorolac (15 or $30 \mathrm{mg}$, according to age and weight) was given during surgical closure. Residual neuromuscular blockade was reversed with glycopyrrolate and neostigmine after assessment of neuromuscular function with train-of-four monitoring.

Blood pressure was assessed noninvasively every 5 minutes throughout the procedure, and heart rate was recorded continuously. In all patients, elevations in mean arterial pressure $(>100)$ and/or heart rate $15 \%$ above baseline (or higher) could be treated with labetalol (10 mg intravenously) and/or propofol (up to a 50-mg bolus), and/ or increased MAC inhaled anesthetics. For the OCA patients, opioids (fentanyl $1-2 \mu \mathrm{g} / \mathrm{kg}$, or Dilaudid up to $2 \mathrm{mg}$ total) were permitted.

\section{Surgical Protocol}

All procedures were performed by a single surgeon (S.A.Q.) with extensive experience in MIS technique. After induction of general anesthesia, patients were positioned prone on a radiolucent table with Wilson frame. After surgical preparation with betadine and ChloraPrep, the SpineMask (Stryker Navigation), a noninvasive, adhesive stereotactic tracker was applied to the skin overlying the operative site, and an intraoperative 3D fluoroscopic spin was performed using a Ziehm Vision RFD 3D C-arm (Ziehm Imaging Inc.). In the event that there were concerns regarding the accuracy of the 3D navigation intraoperatively, traditional fluoroscopic MIS techniques were utilized.

A paravertebral incision was made, extending between the cephalad and caudad pedicles on the symptomatic side. Working through the paravertebral incision, soft-tissue dilators were sequentially introduced to allow an 18$\mathrm{mm}$ tubular retractor to be docked at the lamina overlying the pathological disc level. A laminotomy was performed under microscopic visualization using a high-speed burr, exposing the ligamentum flavum, which was removed with Kerrison rongeurs and providing access to the disc space in cases in which a microdiscectomy was required. The tubular retractor was then adjusted to allow for medial access, and decompression of lamina and ligamentum flavum was performed on the contralateral facet via a singlesided approach. Thrombin-impregnated matrix was used to obtain hemostasis, and the retractor was removed deliberately to ensure hemostasis of the paraspinal soft tissues at the conclusion of the procedures. All patients received subcutaneous infiltration of $10 \mathrm{ml}$ of $0.25 \%$ Marcaine after fascial closure and immediately prior to skin closure.

\section{Postoperative Care}

Pain control, level of alertness, and vital signs were monitored in the PACU. Patients were permitted to drink and eat after recovery from anesthesia. According to our ERAS pathway, patients with reported NRS scores $\leq 4$ should be treated with nonopioid analgesics (acetaminophen, ketorolac, gabapentin, and/or nonpharmacotherapies, including ice, distraction, and position changes); for those with NRS scores 5-7, patients may receive two 50-mg doses of tra-
TABLE 1. Summary of demographic data

\begin{tabular}{|c|c|c|c|}
\hline Variable & OFA & OCA & $p$ Value \& Test \\
\hline Patients, no. & 18 & 18 & \\
\hline $\begin{array}{l}\text { Mean age } \pm \text { SEM, } \\
\text { yrs }\end{array}$ & $61.5 \pm 18.92$ & $60.14 \pm 15.4$ & NS, paired t-test \\
\hline M/F ratio, no. & $10: 8$ & $10: 8$ & $\begin{array}{l}\text { NS, McNemar's } \\
\text { test }\end{array}$ \\
\hline $\begin{array}{l}\text { Mean BMI } \pm S D, \\
\quad \mathrm{~kg} / \mathrm{m}^{2}\end{array}$ & $26.5 \pm 3.40$ & $26.9 \pm 5.0$ & NS, paired t-test \\
\hline ASA class, no. & & & $\begin{array}{l}\text { NS, Wilcoxon } \\
\text { signed-rank }\end{array}$ \\
\hline I & 4 & 4 & \\
\hline II & 13 & 13 & \\
\hline III & 1 & 1 & \\
\hline Smoking status, no. & & & NS, paired t-test \\
\hline $\begin{array}{l}\text { Current (at time } \\
\text { of op) }\end{array}$ & 0 & 1 & \\
\hline Former & 3 & 3 & \\
\hline Never & 1 & 14 & \\
\hline $\begin{array}{l}\text { Baseline opioid } \\
\text { status }\end{array}$ & & & NS, paired t-test \\
\hline Tolerant & 7 & 5 & \\
\hline Naive & 11 & 13 & \\
\hline No. of comorbidities & & & $\begin{array}{l}\text { NS, Wilcoxon } \\
\text { signed-rank }\end{array}$ \\
\hline DM & 1 & 1 & \\
\hline Hypertension & 8 & 10 & \\
\hline Hyperlipidemia & 9 & 8 & \\
\hline OSA & 2 & 2 & \\
\hline Procedure, no. & & & $\begin{array}{l}\text { NS, Wilcoxon } \\
\text { signed-rank }\end{array}$ \\
\hline Microdiscectomy & 12 & 10 & \\
\hline Laminectomy & 6 & 8 & \\
\hline No. of levels, no. & & & $\begin{array}{l}\text { NS, Wilcoxon } \\
\text { signed-rank }\end{array}$ \\
\hline 1 & 15 & 14 & \\
\hline 2 & 2 & 3 & \\
\hline 3 & 1 & 1 & \\
\hline $\begin{array}{l}\text { Mean duration of op } \\
\pm \text { SEM, mins }\end{array}$ & $56.45 \pm 10.82$ & $61.45 \pm 14.66$ & NS, paired t-test \\
\hline $\begin{array}{l}\text { Mean time to recov } \\
\text { from anesthesia } \\
\pm \text { SEM, mins }\end{array}$ & $22 \pm 1.5$ & $24 \pm 1.4$ & NS, paired t-test \\
\hline
\end{tabular}

$\mathrm{BMI}=$ body mass index; $\mathrm{DM}=$ diabetes mellitus; $\mathrm{NS}$ = not significant; OSA = obstructive sleep apnea; recov = recovery.

madol if needed; and for those with NRS scores 8-10, patients may receive a 5-mg oxycodone. Further escalation of opioids requires assessment by the anesthesiologist. PONV are treated with metoclopramide (10 mg intravenously) or ondansetron $(4 \mathrm{mg})$. Patients with refractory PONV are prescribed scopolamine (1.5 mg transdermally). Readiness for discharge from the PACU is determined when patients achieved a modified Aldrete score $\geq 9 .{ }^{20}$ 
Total Opioid Consumption

(Oral Morphine MEQs)

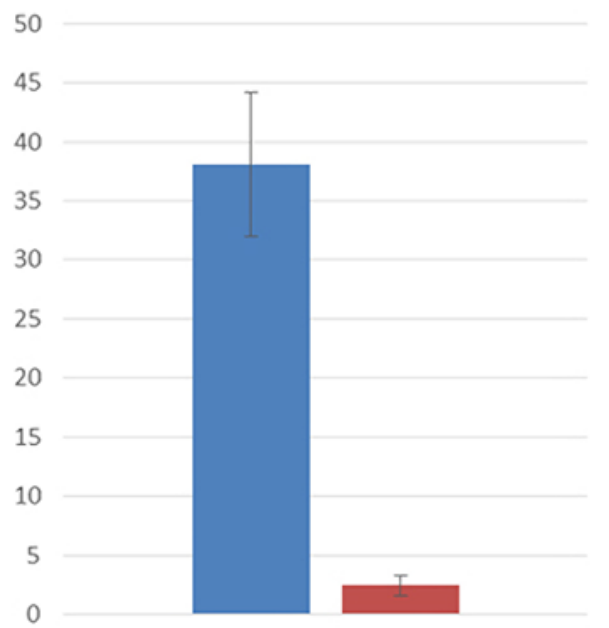

PACU Opioid Consumption

(Oral Morphine MEQs)

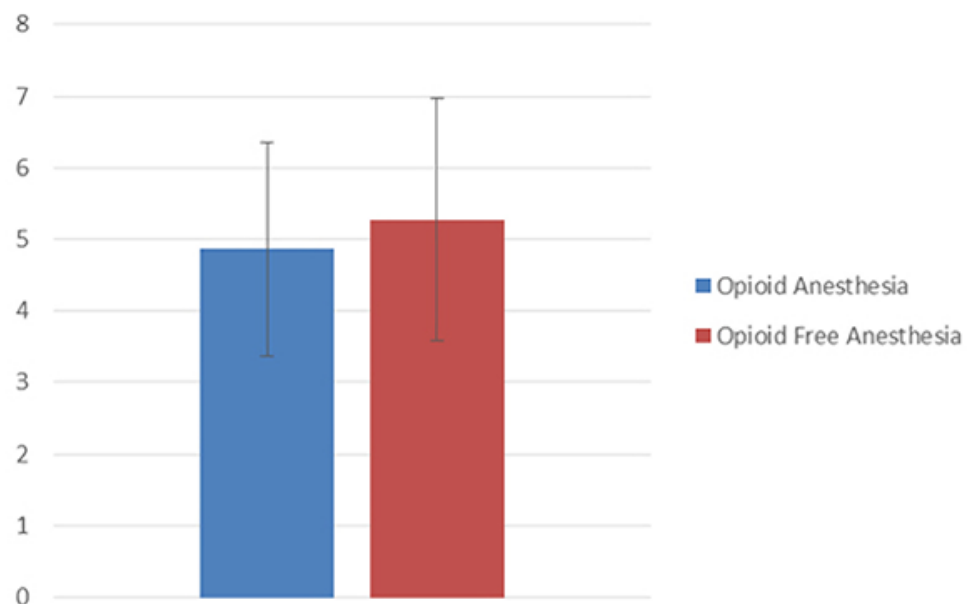

FIG. 1. Left: Total perioperative opioid consumption was significantly greater in the OCA (opioid anesthesia) group (mean \pm SEM $38.125 \pm 6.11$ OMEs) than in the OFA group ( $2.43 \pm 0.86$ OMEs). Right: There was no difference in PACU opioid consumption between OCA (4.86 $\pm 1.5 \mathrm{OMEs})$ and OFA ( $5.28 \pm 1.7 \mathrm{OMEs})$ groups.

\section{Data Collection}

Data on demographics, intraoperative metrics, and recovery were collected and included age, sex, ASA class, smoking status, preoperative opioid status (naïve or tolerant), duration of surgery, time to recovery from anesthesia (defined as the interval from the end of surgery to the transition of care from the anesthesiologist to the PACU nurse), NRS scores, opioid consumption, and LOS (defined as the time between the end of surgery until readiness for discharge from the PACU). Opioid consumption was converted to morphine milliequivalents (mEq).

\section{Statistical Analysis}

The primary outcome variable was total opioid consumption during the admission. Secondary outcomes were PACU opioid consumption, NRS scores (initial scores on arrival to PACU and worst scores), time to anesthetic recovery, and LOS. As a pragmatic study, we did not calculate a sample size requirement to show a significant difference in the primary outcome. Results are presented as mean \pm SEM for normally distributed data, and median and IQR for skewed data. A Mann-Whitney U-test was used to assess LOS. Student t-tests were calculated to compare discreet data (NRS score and opioid consumption). A Pearson product-moment correlation coefficient was computed for associative relationships between pain scores and opioid use in the PACU. For patient demographics, a paired t-test was used to compare continuous numeric data, McNemar test for nominal data, and the Wilcoxon signed-rank test for ordinal data.

\section{Results}

Patient characteristics and perioperative data are detailed in Table 1. Baseline characteristics and intraoperative metrics were not significantly different between groups.
Total mean perioperative opioid consumption was significantly higher in the OCA group (38.13 \pm 6.11 OMEs) compared to the OFA group $(2.43 \pm 0.86$ OMEs; $t(70)=$ $5.8, \mathrm{p}<0.001$; Fig. 1). Total opioid consumption in the PACU was not significantly different between the two cohorts (OCA 4.86 \pm 1.5 ; OFA $5.28 \pm 1.7$; $\mathrm{t}(34)=-0.18$, $\mathrm{p}>$ 0.05 , not significant).

Initial mean NRS scores were slightly higher in the OFA cohort $(1.0 \pm 0.46)$ than the OCA cohort $(1.6 \pm 0.69)$; however, these results were not statistically significantly different $(\mathrm{t}(34)=0.80, \mathrm{p}>0.05$, not significant; Fig. 2). The worst mean NRS scores in the PACU (OCA $2.58 \pm$ 0.73 ; OFA $2.55 \pm 0.70$ ) were also not significantly different $(t(34)=0.16, p>0.05)$ between the two groups (Fig. 2).

A frequency distribution of NRS scores in the PACU was remarkable for $64 \%$ (23/36) of the total cohort rating their worst pain as $\leq 3$ and $13 \%(3 / 23)$ of patients with reported NRS scores $<4$ receiving an opioid analgesic (Fig. 3). Five patients received intravenous opioids in the PACU: 4 patients were given $0.5 \mathrm{mg}$ hydromorphone (for worst reported NRS scores of 6, 8, 8, and 9), and 1 patient was given $1 \mathrm{mg}$ hydromorphone (for worst reported NRS score of 5). Overall, there was a strongly positive correlation between higher NRS scores and opioid administration/consumption in the PACU for both OFA $(r=0.82)$ and OCA $(r=0.77$; Fig. 3$)$ groups.

The median LOS was 37 minutes shorter for patients who received OFA (237 minutes [IQR 174-312 minutes]) compared to OCA (274 minutes [IQR 204-403 minutes]; Fig. 4). However, this was not a statistically significant difference $(\mathrm{U}(34)=123, \mathrm{z}=1.22, \mathrm{p}>0.05)$.

The ERAS pathway included 19 standard elements divided into pre- (5 elements), intra- (10 elements), and postoperative (4 elements) care (Fig. 5). Overall pathway compliance was $91.4 \%$. Use of intraoperative opioid agents was tracked separately and, as expected, was administered 


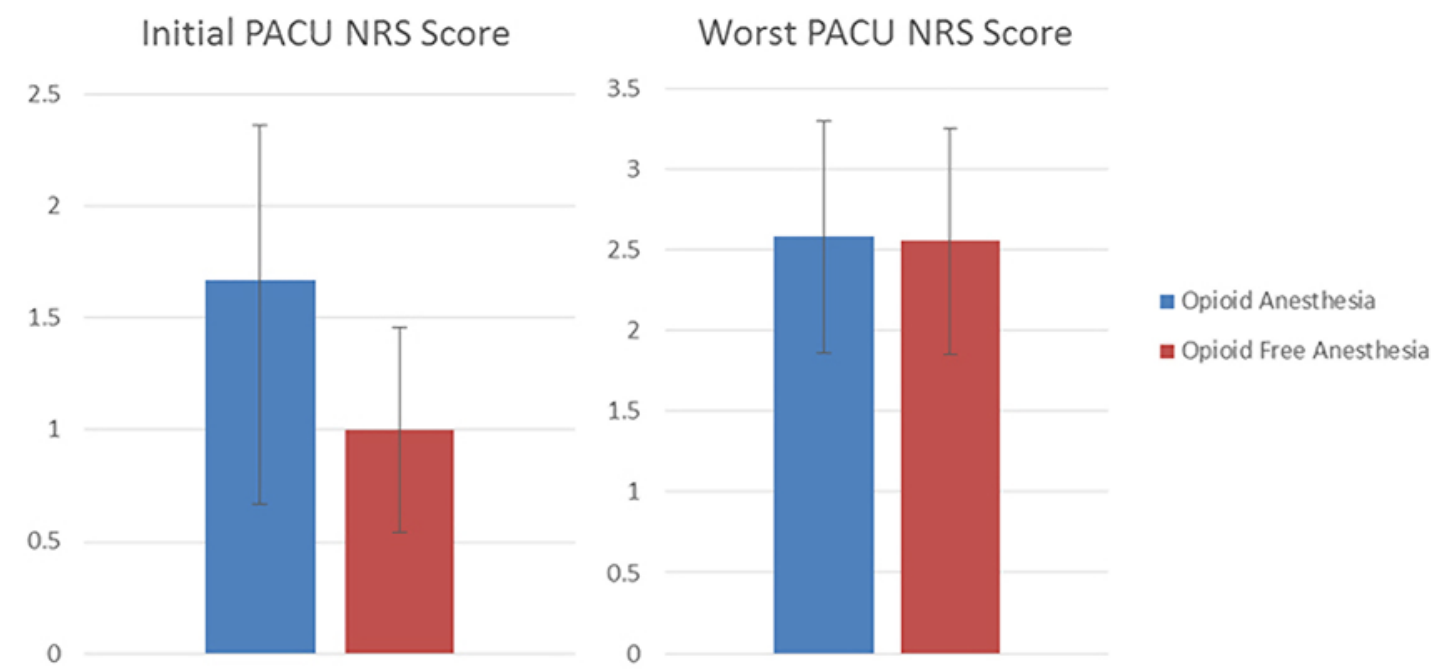

FIG. 2. Left: No difference in PACU initial NRS scores between the groups (OCA $1.6 \pm 0.69$; OFA $1.0 \pm 0.46)$. Right: No difference in worst PACU pain scores between the groups (OCA $2.58 \pm 0.73$; OFA $2.55 \pm 0.70$ ).

to $50 \%$ of patients. Items with the lowest compliance $(<$ $80 \%$ ) were maintenance of intraoperative normothermia (72.2\%), early physical therapy (within 90 minutes of PACU arrival; 75\%), and PACU opioid administration according to pathway and NRS criteria (77.7\%). Components with the highest compliance were MIS approach (100\% of cases, with no conversion to open surgery), and use of multimodal anesthetic (100\%) and analgesic (100\%) agents. An analysis of compliance by phase of care revealed the highest compliance with ERAS process elements was achieved intraoperatively (mean $94.71 \% \pm 2.88 \%$ ), and the lowest compliance was during the postoperative phase of care (mean $85.4 \% \pm 5.7 \%$ ) (Fig. 5).

\section{Discussion}

MMA and ERAS pathways of care have become standard tools for minimizing opioid use and related side effects and improving outcomes after orthopedic surgery. ${ }^{28,29,30,34}$ In contrast, relatively little attention has been paid to the contribution that OFA may make to achieving the same goals. Although gaining prominence in other surgical subtypes, procedure-specific data for the efficacy of OFA is not yet in evidence for spine surgery. Kim et al. described the use of OFA in a patient undergoing a 2-level posterior lumbar fusion; they used dexmedetomidine and lidocaine infusions and concluded possible improved analgesia in the first 24 hours after surgery. ${ }^{14}$ A second report described a fully opioid-free anesthetic and analgesic regimen in a patient undergoing multilevel thoracolumbar spine fusion, featuring erector spinae plane block and comprehensive MMA.

Given the successes of MMA within ERAS pathways, we asked if an OFA technique could provide adequate anesthesia and analgesia while minimizing total opioid con-

\section{Frequency Distribution of Worst NRS Scores in PACU}

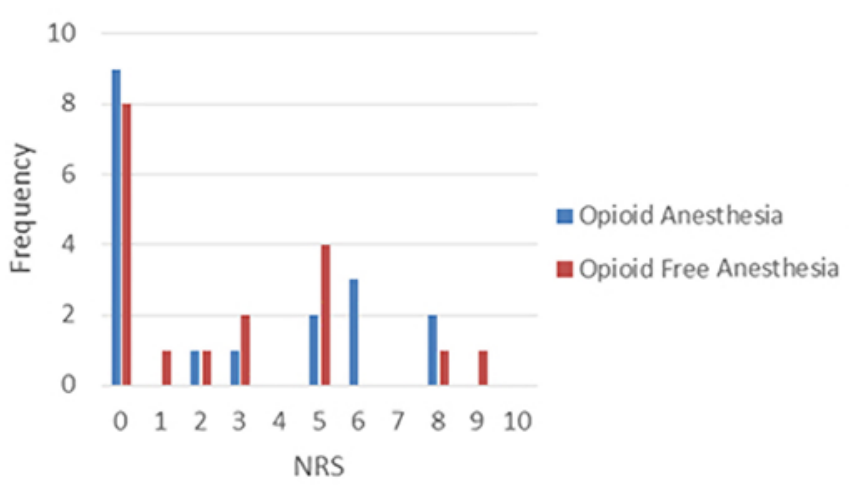

NRS scores and opioid consumption in PACU

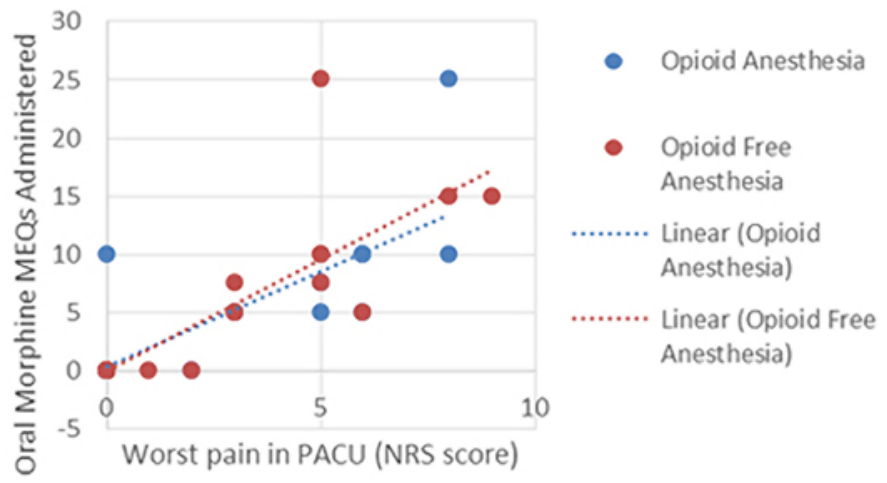

FIG. 3. Left: Twenty-three of 36 patients in the study reported their worst NRS scores during the PACU stay as $\leq 3$. Right: There was an inconsistent relationship between PACU NRS score and opioid consumption: 3 of 23 patients with an NRS score $<4$ received PACU opioids. 
sumption for patients undergoing lumbar decompression. The results of this study suggest that OFA with comprehensive MMA is associated with lower opioid consumption in the perioperative period, without adverse effects on postoperative pain scores, opioid requirements, or recovery. Although not statistically significant, we also showed a clinically significant faster time to readiness for discharge from the PACU in the OFA cohort. We suggest the use of OFA represents a valuable opportunity to limit unnecessary opioid exposure for patients undergoing lumbar decompression within a comprehensive ERAS pathway.

There are many compelling reasons to avoid opioids in any surgical patient. Recognized important side effects include respiratory depression and/or obstruction, nausea, vomiting, constipation and ileus, urinary retention, sedation, and cognitive dysfunction. Recently, data has also linked opioids to increased wound complications and resource consumption after cervical spine surgery ${ }^{11}$ and delayed healing after spinal fusion, in an animal model. ${ }^{12}$ Specific populations of surgical patients that may be even more likely to benefit from OFA include patients with obesity and/or obstructive sleep apnea, or chronic pain..$^{23,35}$ Benefits in the latter population are suggested by the association between opioid exposure and opioid-induced hyperalgesia $(\mathrm{OIH})$. $\mathrm{OIH}$ is a paradoxical increase in pain sensitivity and decrease in pain tolerance following the administration of opioids..$^{18,38}$ Although the existence of and diagnostic criteria for $\mathrm{OIH}$ are controversial, intraoperative remifentanil infusion has been particularly associated as a risk factor for its development. ${ }^{15}$ Accordingly, OFA has been proposed as a strategy to prevent OIH after multiple surgical subtypes, including spinal fusion,,${ }^{14}$ laparoscopic cholecystectomy, ${ }^{3}$ and colorectal surgery. ${ }^{5}$

The appropriateness of OFA as a tool to minimize total opioid consumption is supported by our finding that postoperative pain scores were not negatively affected by withholding intraoperative opioids. On the contrary, the majority of the cohort had no or minimal pain in the PACU. We attribute this finding to high compliance with the ERAS pathway, which emphasizes comprehensive MMA and an MIS approach. ${ }^{32}$ High compliance has been associated with shorter LOS and superior outcomes in large trials of ERAS in other surgical disciplines., ${ }^{2,24}$ In light of this, our findings that $13 \%$ of the cohort received an opioid analgesic in the PACU for an NRS score $\leq 4$ and a further $13 \%$ received intravenous opioid were unexpected. Our ERAS pathway stratifies pain scores and opioid prescribing, and as structured, an NRS score $\leq 4$ is treated with nonopioid analgesics, and escalation to intravenous opioid is contingent on assessment by the anesthesiologist. We do not know the reasons underlying PACU opioid use in these subsets of patients; however, patient and care factors are likely to contribute. Consistent with our findings, recent data suggest noncompliance with ERAS process measures is most commonly observed in the postoperative period..$^{1,25}$ The reasons for noncompliance are complex and have been mainly attributed to medical decision-making in response to changes in patient condition. ${ }^{25}$ However, up to $25 \%$ of postoperative deviations from ERAS interventions are attributed to systems, nursing, and patient preferences for care. ${ }^{9}$ Although difficult to achieve, high compliance with

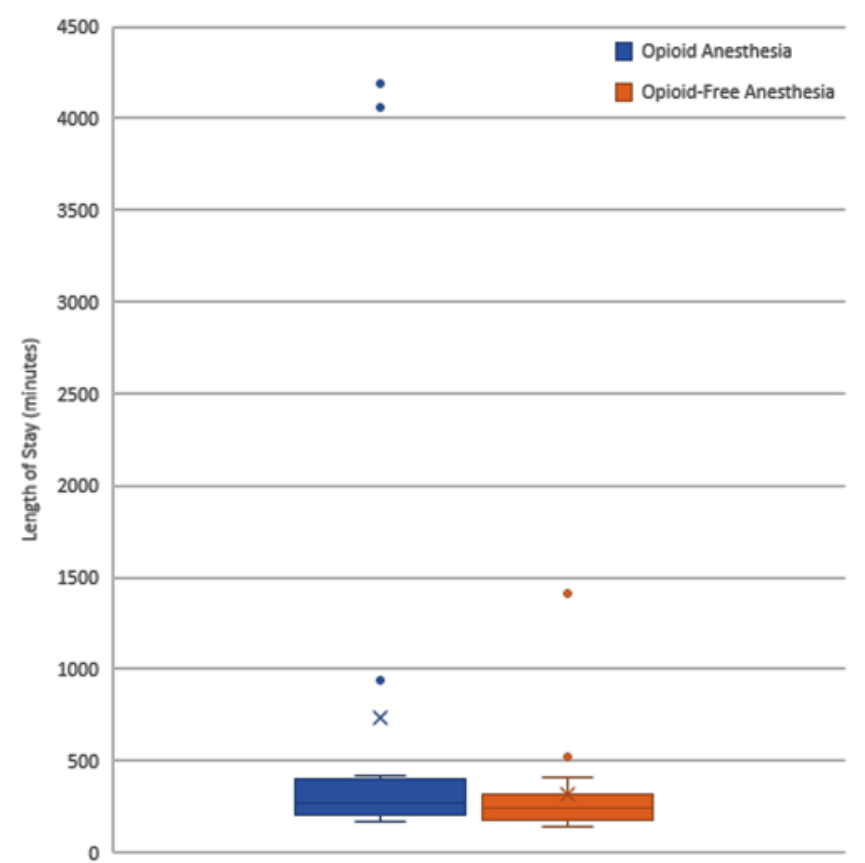

FIG. 4. Median LOS for the OCA group was 274 minutes (IQR 203.75403.25 minutes) and for the OFA group was 237 minutes (IQR 173.75312 minutes). LOS was defined as the time from the end of surgery to the time discharge criteria were met.

postoperative opioid prescribing and pain control may be an important predictor of optimal recovery. A recent retrospective study of an ERAS for colorectal surgery pathway specifically linked high adherence to postoperative opioid prescribing parameters with shorter LOS.10 Barriers to decreasing postoperative opioid at the time of and after discharge persist despite other positive ERAS benefits on opioid consumption: A prospective historical study of prescribing practices before and after implementation of an ERAS protocol for colorectal surgery found little effect on the amount of opioids prescribed at discharge regardless of decreased in-hospital narcotic requirement. ${ }^{5}$ Identifying cultural and organizational barriers to ERAS interventions has long been recognized as vital for pathway success and our results highlight the importance of continual pathway evaluation and education of patients and providers. ${ }^{4}$

A major difference between our OFA regimen and published reports was that we did not include dexmedetomidine. As a centrally acting alpha- 2 adrenergic receptor agonist, dexmedetomidine decreases adrenergic outflow and blunts the sympathetic-adrenal response to surgical stimulation. ${ }^{26}$ These actions promote intraoperative hemodynamic stability, making dexmedetomidine a frequent opioid substitute in OFA.7,15,21 Dexmedetomidine may also have analgesic properties: One meta-analysis concluded that dexmedetomidine reduced early postoperative pain scores and opioid consumption when administered intraoperatively to patients receiving general anesthesia in mixed surgical cohorts. ${ }^{37}$ However, a more recent systematic review of the analgesic benefits of dexmedetomidine for abdominal surgery was less optimistic, concluding that 

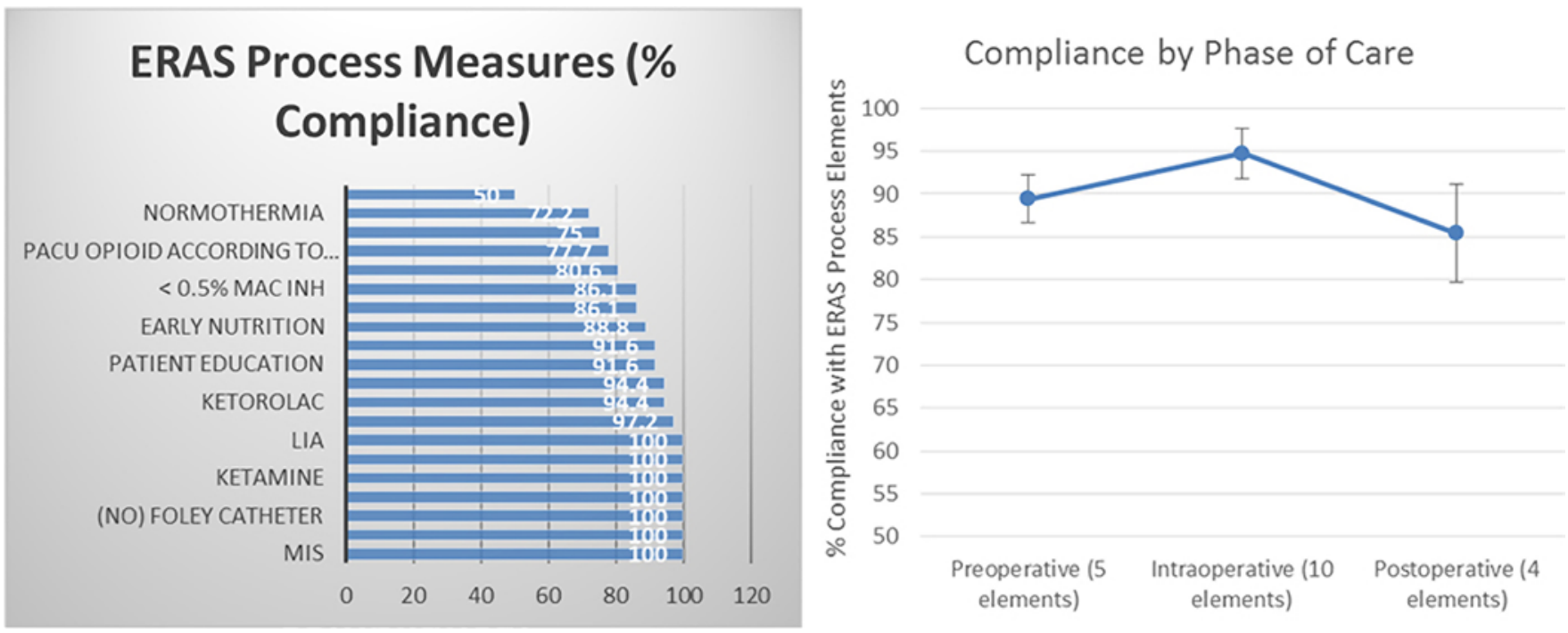

FIG. 5. Left: ERAS-for-spine-pathway compliance. Overall pathway compliance was $91.4 \%$. Right: The highest compliance with ERAS process measures was during the intraoperative phase (mean $94.71 \% \pm 2.88 \%$ ), and the lowest compliance was during the postoperative phase (mean $85.4 \% \pm 5.7 \%$ ). Use of intraoperative opioid (administered to $50 \%$ of the cohort) was excluded from this analysis. LIA = local infiltration analgesia; MAC-INH = minimum alveolar concentration, inhaled anesthetic.

the majority of studies were of mixed quality and most compared dexmedetomidine to placebo. ${ }^{13}$ A meta-analysis of dexmedetomidine as an adjunct in spine surgery confirmed an opioid-sparing effect-both intra- and postoperatively - but failed to find additional benefits, including PONV. ${ }^{36}$ At the time of our investigation, dexmedetomidine was not available in our institution. Although omitted for pragmatic reasons, our results suggest that dexmedetomidine may not be necessary for successful OFA for all surgical subtypes. Future studies should be directed toward clarifying the role of dexmedetomidine as an analgesic adjunct in spine surgery and to defining procedure specific OFA regimens.

This study has several limitations. It was a small study that was not powered to detect significant differences in postoperative pain states between OCA and OFA. The primary aim of this study was to demonstrate the feasibility of OFA within an ERAS MIS lumbar decompression pathway. The pathway has already been implemented at a large, tertiary care orthopedic hospital. As a result, the processes and findings of this study may not be generalizable to nonacademic community settings, locales with a lower volume of procedures, or an institution where minimally invasive techniques are not available. Despite inclusion of a matched cohort, the retrospective study design, small sample size, and absence of power calculation limit the strength of our conclusions regarding the benefits of OFA on recovery. Furthermore, the majority of our patients were classified as ASA class I or II, which limits the application of this protocol in practice settings with a higher patient comorbid burden. Additionally, some important outcomes of interest, such as opiate consumption following discharge, incidence of PONV, and the need for antiemetic medication in the PACU, were not collected and included in this analysis. Despite this limitation, we believe that our study addresses the primary objective of this study, which was to demonstrate the feasibility of OFA as part of an ERAS protocol in minimally invasive spine surgery.

\section{Conclusions}

Here we report the first cohort study of OFA for spine surgery. The results suggest that OFA for MIS lumbar decompression is feasible. Within a comprehensive ERAS pathway emphasizing MMA, OFA did not adversely affect surgical duration, postoperative (rebound) pain, recovery, or LOS. On the contrary, our results suggest the possibility that OFA may be associated with more rapid time to meeting discharge criteria. As a novel paradigm, further studies are required to evaluate OFA and its applicability to other spine surgeries. Additional questions to be answered include how best to monitor intraoperative nociception (particularly for surgeries with higher expected pain burden); which anesthetic and analgesic adjuvants should be included in the regimen; what the indications and contraindications are to OFA; and what the effects of OFA are on long-term outcomes, such as total (postdischarge) opioid use and duration, return to function, and influence on the development of chronic pain. In the case of MIS lumbar decompression, we believe a prospective randomized controlled trial with an OFA regimen including dexmedetomidine would answer many of these questions. Despite these unknowns, in the current setting of the opioid crisis, OFA may represent an additional tool to protect patients from the harms of opioid exposure.

\section{References}

1. Aarts MA, Rotstein OD, Pearsall EA, Victor JC, Okrainec A, McKenzie M, et al: Postoperative ERAS interventions have the greatest impact on optimal recovery: experience 
with implementation of ERAS across multiple hospitals. Ann Surg 267:992-997, 2018

2. Ahmed J, Khan S, Lim M, Chandrasekaran TV, MacFie J: Enhanced recovery after surgery protocols - compliance and variations in practice during routine colorectal surgery. Colorectal Dis 14:1045-1051, 2012

3. Bakan M, Umutoglu T, Topuz U, Uysal H, Bayram M, Ka$\operatorname{dioglu} \mathrm{H}$, et al: Opioid-free total intravenous anesthesia with propofol, dexmedetomidine and lidocaine infusions for laparoscopic cholecystectomy: a prospective, randomized, double-blinded study. Braz J Anesthesiol 65:191-199, 2015

4. Bona S, Molteni M, Rosati R, Elmore U, Bagnoli P, Monzani $\mathrm{R}$, et al: Introducing an enhanced recovery after surgery program in colorectal surgery: a single center experience. World J Gastroenterol 20:17578-17587, 2014

5. Brandal D, Keller MS, Lee C, Grogan T, Fujimoto Y, Gricourt Y, et al: Impact of Enhanced Recovery After Surgery and opioid-free anesthesia on opioid prescriptions at discharge from the hospital: a historical-prospective study. Anesth Analg 125:1784-1792, 2017

6. Brummett CM, Waljee JF, Goesling J, Moser S, Lin P, Englesbe MJ, et al: New persistent opioid use after minor and major surgical procedures in US adults. JAMA Surg 152:e170504, 2017

7. Chin KJ, Lewis S: Opioid-free analgesia for posterior spinal fusion surgery using erector spinae plane (ESP) blocks in a multimodal anesthetic regimen. Spine (Phila Pa 1976) [epub ahead of print], 2018

8. Feld JM, Laurito CE, Beckerman M, Vincent J, Hoffman WE: Non-opioid analgesia improves pain relief and decreases sedation after gastric bypass surgery. Can J Anaesth 50:336-341, 2003

9. Gramlich LM, Sheppard CE, Wasylak T, Gilmour LE, Ljungqvist $\mathrm{O}$, Basualdo-Hammond $\mathrm{C}$, et al: Implementation of Enhanced Recovery After Surgery: a strategy to transform surgical care across a health system. Implement Sci 12:67, 2017

10. Grant MC, Pio Roda CM, Canner JK, Sommer P, Galante D, Hobson D, et al: The impact of anesthesia-influenced process measure compliance on length of stay: results from an Enhanced Recovery After Surgery for Colorectal Surgery cohort. Anesth Analg 128:68-74, 2019

11. Jain N, Brock JL, Phillips FM, Weaver T, Khan SN: Chronic preoperative opioid use is a risk factor for increased complications, resource use, and costs after cervical fusion. Spine J 18:1989-1998, 2018

12. Jain N, Himed K, Toth JM, Briley KC, Phillips FM, Khan SN: Opioids delay healing of spinal fusion: a rabbit posterolateral lumbar fusion model. Spine J 18:1659-1668, 2018

13. Jessen Lundorf L, Korvenius Nedergaard H, Møller AM: Perioperative dexmedetomidine for acute pain after abdominal surgery in adults. Cochrane Database Syst Rev 2:CD010358, 2016

14. Kim DJ, Bengali R, Anderson TA: Opioid-free anesthesia using continuous dexmedetomidine and lidocaine infusions in spine surgery. Korean J Anesthesiol 70:652-653, 2017

15. Kim SH, Stoicea N, Soghomonyan S, Bergese SD: Remifentanil-acute opioid tolerance and opioid-induced hyperalgesia: a systematic review. Am J Ther 22:e62-e74, 2015

16. Koepke EJ, Manning EL, Miller TE, Ganesh A, Williams DGA, Manning MW: The rising tide of opioid use and abuse: the role of the anesthesiologist. Perioper Med (Lond) 7:16, 2018

17. Lam KK, Mui WL: Multimodal analgesia model to achieve low postoperative opioid requirement following bariatric surgery. Hong Kong Med J 22:428-434, 2016

18. Lavand'homme P, Steyaert A: Opioid-free anesthesia opioid side effects: tolerance and hyperalgesia. Best Pract Res Clin Anaesthesiol 31:487-498, 2017
19. Mansour MA, Mahmoud AAA, Geddawy M: Nonopioid versus opioid based general anesthesia technique for bariatric surgery: a randomized double-blind study. Saudi J Anaesth 7:387-391, 2013

20. Marshall SI, Chung F: Discharge criteria and complications after ambulatory surgery. Anesth Analg 88:508-517, 1999

21. Mauermann E, Ruppen W, Bandschapp O: Different protocols used today to achieve total opioid-free general anesthesia without locoregional blocks. Best Pract Res Clin Anaesthesiol 31:533-545, 2017

22. Mulier J, Dekock M: Opioid free general anesthesia, a new paradigm? Best Pract Res Clin Anaesthesiol 31:441-443, 2017

23. Mulier JP: Perioperative opioids aggravate obstructive breathing in sleep apnea syndrome: mechanisms and alternative anesthesia strategies. Curr Opin Anaesthesiol 29:129133,2016

24. Rogers LJ, Bleetman D, Messenger DE, Joshi NA, Wood L, Rasburn NJ, et al: The impact of enhanced recovery after surgery (ERAS) protocol compliance on morbidity from resection for primary lung cancer. J Thorac Cardiovase Surg 155:1843-1852, 2018

25. Roulin D, Muradbegovic M, Addor V, Blanc C, Demartines N, Hübner M: Enhanced Recovery after Elective Colorectal Surgery-reasons for non-compliance with the protocol. Dig Surg 34:220-226, 2017

26. Scholz J, Tonner PH: $\alpha 2$-adrenoceptor agonists in anaesthesia: a new paradigm. Curr Opin Anaesthesiol 13:437-442, 2000

27. Seth P, Scholl L, Rudd RA, Bacon S: Overdose deaths involving opioids, cocaine, and psychostimulants-United States, 2015-2016. MMWR Morb Mortal Wkly Rep 67:349-358, 2018

28. Soffin EM, Gibbons MM, Ko CY, Kates SL, Wick E, Cannesson M, et al: Evidence review conducted for the Agency for Healthcare Research and Quality Safety Program for Improving Surgical Care and Recovery: focus on anesthesiology for total knee arthroplasty. Anesth Analg [epub ahead of print], 2018

29. Soffin EM, Gibbons MM, Ko CY, Kates SL, Wick EC, Cannesson M, et al: Evidence review conducted for the Agency for Healthcare Research and Quality Safety Program for Improving Surgical Care and Recovery: focus on anesthesiology for total hip arthroplasty. Anesth Analg [epub ahead of print], 2018

30. Soffin EM, Gibbons MM, Wick EC, Kates SL, Canneson M, Scott MJ, et al: Evidence review conducted for the Agency for Healthcare Research and Quality Safety Program for Improving Surgical Care and Recovery: focus on anesthesiology for hip fracture surgery. Anesth Analg [epub ahead of print], 2018

31. Soffin EM, Lee BH, Kumar KWC: The prescription opioid crisis: the role of the anesthesiologist in reducing opioid consumption. Br J Anaesth [epub ahead of print], 2018

32. Soffin EM, Vaishnav AS, Wetmore D, Barber L, Hill P, Gang $\mathrm{CH}$, et al: Design and implementation of an Enhanced Recovery After Surgery (ERAS) program for minimally invasive lumbar decompression spine surgery: initial experience. Spine (Phila Pa 1976) [epub ahead of print], 2018

33. Soffin EM, Waldman SA, Stack RJ, Liguori GA: An evidence-based approach to the prescription opioid epidemic in orthopedic surgery. Anesth Analg 125:1704-1713, 2017

34. Soffin EM, YaDeau JT: Enhanced recovery after surgery for primary hip and knee arthroplasty: a review of the evidence. Br J Anaesth 117 (Suppl 3):iii62-iii72, 2016

35. Sultana A, Torres D, Schumann R: Special indications for opioid free anaesthesia and analgesia, patient and procedure related: including obesity, sleep apnoea, chronic obstructive pulmonary disease, complex regional pain syndromes, opioid 
addiction and cancer surgery. Best Pract Res Clin Anaesthesiol 31:547-560, 2017

36. Tsaousi GG, Pourzitaki C, Aloisio S, Bilotta F: Dexmedetomidine as a sedative and analgesic adjuvant in spine surgery: a systematic review and meta-analysis of randomized controlled trials. Eur J Clin Pharmacol 74:1377-1389, 2018

37. Wang X, Liu N, Chen J, Xu Z, Wang F, Ding C: Effect of intravenous dexmedetomidine during general anesthesia on acute postoperative pain in adults: a systematic review and meta-analysis of randomized controlled trials. Clin J Pain 34:1180-1191, 2018

38. Weber L, Yeomans DC, Tzabazis A: Opioid-induced hyperalgesia in clinical anesthesia practice: what has remained from theoretical concepts and experimental studies? Curr Opin Anaesthesiol 30:458-465, 2017

39. Wick EC, Grant MC, Wu CL: Postoperative multimodal analgesia pain management with nonopioid analgesics and techniques: a review. JAMA Surg 152:691-697, 2017

40. Ziemann-Gimmel P, Goldfarb AA, Koppman J, Marema RT: Opioid-free total intravenous anaesthesia reduces postoperative nausea and vomiting in bariatric surgery beyond triple prophylaxis. Br J Anaesth 112:906-911, 2014

\section{Disclosures}

Dr. Albert currently receives grant/research support from PCORI, ISSG, NIH, and Alan L. and Jacqueline B. Stuart Spine Research Center; receives royalties from Zimmer Biomet, DePuy Synthes, NuVasive, JP Medical Publishers, Saunders/Mosby-Elsevier, and Thieme; receives consulting fees from Facet Link, DePuy, Biomet, NuVasive, and Gentis (medical advisory board); has stock/ investment interests in Vital 5, Bonovo Orthopedics Inc., Biomerix, InVivo Therapeutics, Spinicity, Crosstrees Medical, Paradigm Spine LLC, Invuity, Gentis, ASIP, PMIG, Vertech, and Pioneer; has a leadership role at Scoliosis Research Society; is an employee of the Hospital for Special Surgery and Weill Cornel Medical Center; and holds US patents (US7,955,358 B2 and US9717541

B2). Dr. Qureshi currently receives consulting fees from ZimmerBiomet, Stryker Spine, Globus Medical, Inc.; has shareholder interest in Avaz Surgical and Vital 5; receives royalties from RTI, Zimmer-Biomet, and Stryker Spine; and is a board member of the Minimally Invasive Spine Surgery Group and Healthgrades.

\section{Author Contributions}

Conception and design: Soffin, Wetmore, Vaishnav, Albert, Qureshi. Acquisition of data: Soffin, Wetmore, Beckman, Vaishnav, Albert, Gang. Analysis and interpretation of data: Soffin, Vaishnav. Drafting the article: Soffin, Wetmore, Beckman, Sheha, Qureshi. Critically revising the article: Soffin, Sheha, Albert, Qureshi. Reviewed submitted version of manuscript: all authors. Statistical analysis: Soffin. Administrative/technical/material support: Soffin, Beckman, Sheha, Vaishnav, Albert, Gang, Qureshi. Study supervision: Soffin, Vaishnav, Albert, Qureshi.

\section{Correspondence}

Ellen M. Soffin: Hospital for Special Surgery, New York, NY. soffine@hss.edu. 